\section{Inside knowledge}

\section{Interpreting Minds: The Evolution of a Practice \\ by Radu Bogdan}

MIT Press: 1997. Pp. 304. \$35, £29.50

\section{Andrew Whiten}

For most of us, reading others' minds is an everyday accomplishment: not only may we be concerned with what others think of us, but our thoughts may even extend to what they think we think of them. How and what we may know of other minds has long exercised philosophers, but the past decade has shown that our everyday mind-reading can be examined scientifically.

The results have been most prominent in the field of developmental psychology, which has seen the growth of a gigantic literature on what has come to be called the child's 'theory of mind'. Other researchers have attempted to reconstruct the evolution of such abilities by studying primates, following the experiments pioneered by David Premack and Guy Woodruff to discover whether the chimpanzee has a theory of mind.

It is not surprising that philosophers have found this work of interest. What is more, there has recently been real dialogue between philosophers of mind and experimentalists, seriously enriching the scope of the scientific enterprise. Indeed, some philosophers have become so excited as to begin experimenting themselves. The philosopher Radu Bogdan is not one of these, but he has done an impressive job of assimilating the new empirical literature in all its breadth, and his book is a work of psychology as much as philosophy.

In his own words, his task has been to "get to the roots" of this extraordinary competence of ours. He pursues this in part by charting in considerable detail accomplishments identified from infancy to middle childhood, together with their hypothesized functions and underlying cognitive procedures.

This is not a book to read as an introduction to the field: it will be challenging even for those already familiar with the primary literature. But for the growing 'theory of mind' community, it provides a distinctive analysis of the most fundamental questions about how we interpret the actions of others.

This is important because, despite the outpourings of experimental results, the field still lacks any deep appreciation of the nature of what 'mind-reading' can be, given the nature of mind: we are not telepathists, so what on earth can be going on when we act as if we are?

Bogdan's unique contribution is timely and welcome: it extends the dialogue between philosophers and cognitive scientists beyond recent constrained debates about whether 'theory of mind' is really theory-like or whether it operates by using the self's mind to simulate others; and, no less important, it goes beyond taking evolutionary psychology seriously, to put it at the heart of the analysis.

But what Bogdan has to say can also be problematic. At many points I found the text impenetrable, over-abstract or unconvincing in its conclusions; as a margin-scribbler, I have seldom added so many question marks, including those of the "meaning what?", "who says?" and "how do we know?" variety.

Bogdan argues, correctly I think, that the practical use of mind-reading has not been adequately addressed in developmental psychology. But his argument — that recognizing the evolutionary history of its practical benefits reduces the need to explain the abilities of natural psychologists as mindreading (he prefers to talk only of "interpretation") - is insufficiently clear or persuasive. I do not see practical use and the recognition of states of mind as being in opposition; and evolutionary theorists have neglected neither.

Nevertheless, Bogdan's analysis should provoke thoughtful consideration and discussion across philosophy, cognitive science and ethology, in which the nature of mindreading is treated with a much-needed new depth and breadth.

Andrew Whiten is at the School of Psychology, University of St Andrews, St Andrews, Fife KY16 9JU, UK.

\section{Ways of seeing}

\section{Art and Representation: New Principles in the Analysis of Pictures}

by John Willats

Princeton University Press: 1997. Pp. 394

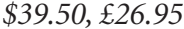

\section{John M. Kennedy}

John Willats argues that picture perception can be broader in scope than real-world perception. Take outline drawings, where a line typically shows the edge of a surface of an object. Curiously, the line usually has two borders yet they show only one edge: that is, the number of features of the line on the picture surface is not the number of features in the depicted scene.

Also, the features of the line can have more than one function. We may, for instance, draw a line around a patch of the picture surface merely to mean "this patch one of nearly 400 photographs featured in A Yellowstone Album (Roberts Rinehart, \$19.95), which commemorates the park's 125th anniversary. 
stands for a facet of an object". Willats describes this as using the "region" on the surface to stand for a face of the referent.

What's more, we sometimes use the region to stand for the whole object - as a "volume". If someone making a line sketch wants us to take the region to be relevant, the line serves only as a convenient device to delimit the region - it is not meant to stand for an edge. Similarly, a Chinese brush painting of a furry animal may have a region of ink standing for the animal's body, but the edges of the inked patch are not to be taken as a detailed rendition of the animal's contours. And a line around the patch can be dotted like an outline in a mosaic from Ravenna.

On a misty morning, or after a celebration, we may often have seen a real edge of an object such as Tony Blair as slightly blurred, but we have never seen it as really composed of dots. Pictorial elements are more varied than real-world elements.

Willats contends that children beginning to draw can probably produce an elongated line and an oval shape. With these two picture-primitives, they can successfully draw only things extended mostly in one dimension (arms, stems, people, bottles) or roughly equally in three dimensions (houses, cars, elephants). Relying on this 'extendedness', young children have trouble drawing objects intermediate in shape between rocks and stems - ones extended in two dimensions mostly, such as 'slabs' like books, doors, plates and coins.

When we become proficient at drawing, particular junctions of lines have particular meanings. An L-junction can show the sharp, rectangular corner of a book. A Tjunction, usefully, can show one surface edge overlapping another. The cross-bar of the $\mathrm{T}$ shows the foreground edge and the stem shows the edge of the rear surface. And the end of a line can show a fold in a surface diminishing to nothing.

As well as outline drawings, Willats describes the picture-primitives of rounded forms (in pictures by Paul Klee), silhouettes (used in early Greek vases) and crosshatchings of various kinds, including ones where shading is used to suggest light and shadow (in nineteenth-century technical drawings of machinery, for example) and ones where the orientations of the hatches indicate the orientation of the depicted surface ('bracelet' shading, used in sixteenthcentury engravings, for example).

Real objects do not come crosshatched, so once again picture perception cannot be predicted directly from real-world perception.

We often draw long objects such as nearby buildings using parallels to show the receding sides, even though the actual sides of the buildings make a smaller and smaller angle at our eye with distance. We find these parallel projections quite acceptable even if the picture's projection at our eye is a far cry from nature's projection.

But we have limits to the range of projections that appear quite acceptable. The religious art of the Byzantine empire took advantage of vision's limits, artfully relying on divergent perspective - diverging lines on the picture standing for receding parallel sides of artefacts - to create anomalies. Willats stresses that these were meant to strike the Orthodox viewer as unrealistic meant deliberately to be incoherent and self-contradictory.

In practice, we often make use of varied geometries of visual representation, exploiting the wide capacities of the visual system. Children do not simply draw by copying the shapes of objects as if making an imprint of a face of the object.
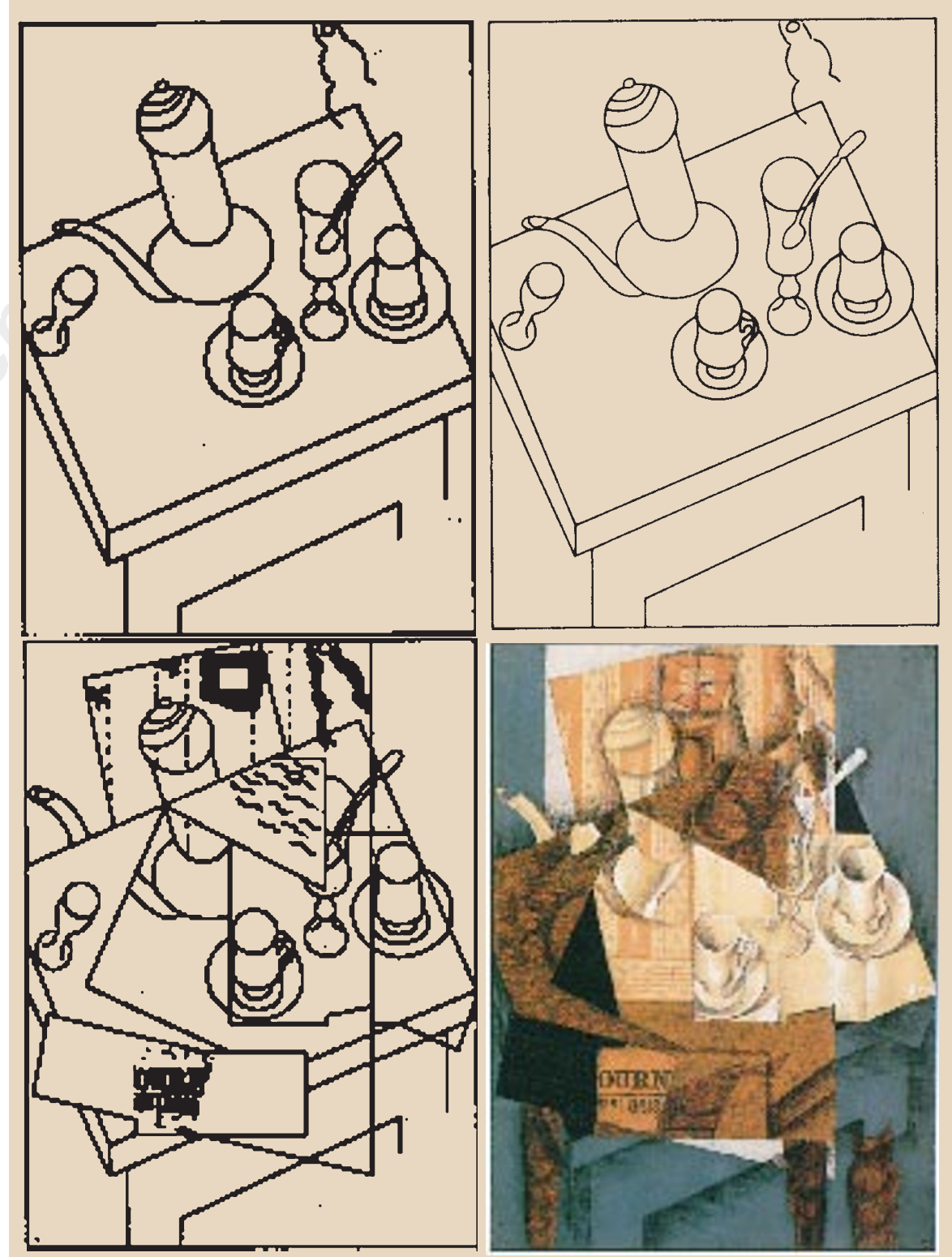

Making a meal of it: illustration from the cover of Art and Representation, showing the application of Willats's analysis to Breakfast by Juan Gris (1914; in the Museum of Modern Art, New York).

Willats explains that the child uses various geometries in the development of drawing prowess. A three-year-old is concerned with what is a detached object, what is enclosed in what and what touches what. These are features of topology, the 'rubbersheet' geometry in which connections are preserved. The child attending to topological features will draw what is inside an object in the real world inside the region in the picture that stands for that object.

Only later in development will the child begin to copy the relevant shape of the object, so a square will stand for a cube. This is a shape produced by a similarity geometry, or orthogonal projection. But, Willats strikingly claims, at this stage the square stands for the entire cube. His evidence is that, if we give the child a cube with differently coloured faces, the child will colour 
the square with differently coloured patches, one for each of the cube's faces.

I hesitate to accept his argument here, since the child may mean "this is a face of the object, and here are some colours it can have". I also balk at his proposal that the child makes much use of topology, since an $\mathrm{X}$ and an I are the same in topology, neither one enclosing a space, but the two are distinct for kindergarten children. Willats admits that children must add some "shape modifiers" to topology.

Willats points out that children often produce pictures that have traditionally been described as using one primary geometry of depiction - parallel projection that projects faces of the object onto the picture surface. If the parallel lines of the projection are at an oblique to the picture surface, side faces can be projected as well as front faces.

But, he argues interestingly, the child actually arrives at these drawings by a short circuit — a 'secondary' geometry — and not by imagining the full procedures of parallel or polar projection - 'primary' geometry of projection.

He writes that many of the puzzling shapes along the route of development "can be explained by supposing that they are derived from internal object-centred descriptions by the application of rules based on secondary rather than primary geometry".

A secondary-geometry rule the typical child uses to produce folded-out forms is "draw receding sides of objects attached to the front face, and with their true shapes". To suggest depth, the child may draw only the front face and one receding face.

A slightly more sophisticated rule in secondary geometry is "show the receding sides of a building by parallel lines at an acute angle to the horizontal". This rule allows the child to draw the front, the top and one side of an object - all the parts that can face a single vantage point. An advanced secondary-geometry rule is "make the lines for a roof's edges, receding into the distance, slope downwards in the picture" and "make the lines for any steps at the base of a building, also receding into the distance, slope upwards".

Willats argues that many pictures from classical times and thirteenth- and fourteenth-century Italy obey these rules about slopes. The rule many children eventually come to is "if the side recedes, draw it using converging lines".

In sum, Willats offers clear applications of his ideas to an immense diversity of pictures from various sources. His tour de force will be the jumping-off point for most picture-perception researchers in the near future. But what are those jumping-off points? The contentious issues can be divided into two camps, developmental and cross-cultural.
Willats' theory provides the child aged 6 to11 with secondary geometry, deriving pictures from object-centred knowledge and rules about receding faces, but it leaves no clear place for perspective effects such as foreshortening. This allows Willats to predict that the child will transform receding shapes such as squares into diamonds or rhombic forms. But he cannot predict consistent foreshortening and in particular the actual amount of foreshortening the child will use.

Western children of 8,9 and 10 do use foreshortening, reliably, and in particular they foreshorten receding sides of cubes to about 70 per cent of the size of the lines standing for the front edges of the cube.

The same limitation obtains when Willats examines the widespread use of oblique projection in pictures from China and Japan over the centuries. For example, the receding sides of screens depicted in a twelfth-century Chinese picture are also foreshortened about 70 per cent. It is likely that view-centred processes for making pictures are indicated by the 70 per cent foreshortening we see in these pictures from diverse sources. I suggest we need a theory of visual inspection, not secondary geometry, to explain why foreshortening crops up so regularly.

Willats' book bids fair to make the study of 'pictorial cues' central to vision science. It will shape debate about drawing development for many years.

John M. Kennedy is in the Life Science-Psychology Department, University of Toronto, 1265

Military Trail, Scarborough, Ontario M1C 1A4, Canada.

\section{Metallurgy - and other animals}

\section{Stuff: The Materials the World is Made of}

by Ivan Amato

BasicBooks: 1997. Pp 304. \$25

\section{Paul Calvert}

Metallurgy used to refer simply to the extraction of metals, but the development of new alloys and of the electron microscope led to a science of the structure of metals. Metallurgy remains a proper discipline, with fundamental theories, methods and boundaries.

Things fell apart when the subject extended to become materials science, with the growing use of polymers, ceramics, glasses and composites in engineering. The problem is that all materials are different and we no longer have a discipline.

Ivan Amato does an excellent job of surveying the field without getting trapped into improvising structure where there is none. He starts historically and takes us from flint-knapping to the formation of the first materials laboratories and departments in the 1960s.

He then draws on his experience as a science journalist visiting the US Materials Research Society (MRS) meetings. He makes an analogy with the Serengeti National Park in Tanzania, in the sense that this large area is populated by small herds of scientists, grazing together and wandering across the conceptual landscape. Since this prompts one to identify the rhinos, ostriches, hyenas and elephants among one's colleagues, the book is worth the price for this analogy alone.

The particular herds that Amato selects - he politely refers to them as tribes - are synthetic diamond, biomimetic materials, smart materials, fullerenes and gallium arsenide.

Two final chapters focus on particular stories: Federico Capasso at Lucent Technologies and the quantum cascade laser, followed by Greg Olson at Northwestern University and steel microstructures. This gives us a view of the individual antelope in the herd.

Most materials departments are called Materials Science and Engineering and sit in engineering colleges. But there are very few true materials engineers who actually design a system to do a particular job in a defined framework. Most work in researchand-development laboratories and cover the range from study-and-explain to enlightened trial-and-error.

So this is not a science as physics is, and it is not engineering as chemical engineering is. If there is a parallel science, it is biology in that it involves the study, and now engineering, of a collection of things that happen to exist.

The MRS, founded in 1973, reflects the Serengeti analogy. It is large but was designed to be fairly unstructured so it could respond to, rather than guide, the field. It has steered away from qualifications, accreditation and representation.

An interesting omission from Stuffis any reference to teaching. Most university materials laboratories have many undergraduate research assistants. The national laboratories carry out a large fraction of materials research and have a large population of graduate students borrowed from universities. I suspect we believe more in apprenticeship than in book-learning.

Materials science is now grown up. It may settle down and become a discipline or continue in fruitful anarchy. Stuff gives a good portrait of its youth.

Paul Calvert is in the Department of Materials Science and Engineering, University of Arizona, 4715 E. Fort Lowell Road, Tucson, Arizona 85712, USA. 